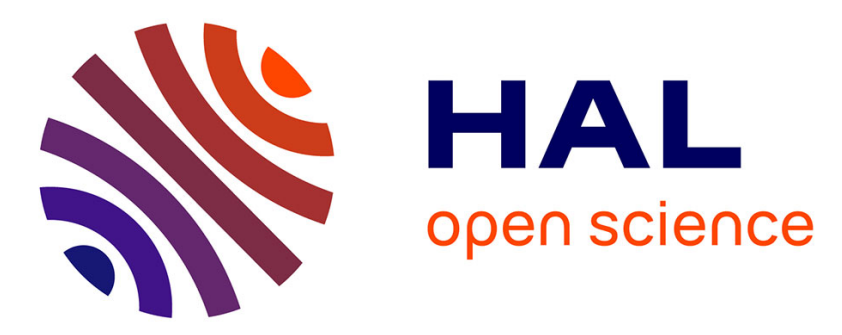

\title{
Sharing the Global Benefits of Finite Natural Resource Exploitation: A Dynamic Coalitional Stability Perspective
}

Stéphane Gonzalez, Fatma Rostom

\section{To cite this version:}

Stéphane Gonzalez, Fatma Rostom. Sharing the Global Benefits of Finite Natural Resource Exploitation: A Dynamic Coalitional Stability Perspective. 2019. halshs-02430751

\section{HAL Id: halshs-02430751 \\ https://shs.hal.science/halshs-02430751}

Preprint submitted on 7 Jan 2020

HAL is a multi-disciplinary open access archive for the deposit and dissemination of scientific research documents, whether they are published or not. The documents may come from teaching and research institutions in France or abroad, or from public or private research centers.
L'archive ouverte pluridisciplinaire HAL, est destinée au dépôt et à la diffusion de documents scientifiques de niveau recherche, publiés ou non, émanant des établissements d'enseignement et de recherche français ou étrangers, des laboratoires publics ou privés. 
UMR 5824

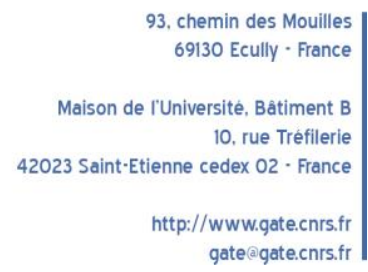

WP 1937 - December 2019

\title{
Sharing the Global Benefits of Finite Natural Resource Exploitation: A Dynamic Coalitional Stability Perspective
}

\author{
Stéphane Gonzalez, Fatma Zahra Rostom
}

\begin{abstract}
:
The article explores the implications of natural resource scarcity in terms of global cooperation and trade. We investigate whether there exist stable international long-term agreements that take into account the disparities between countries in terms of geological endowments and productive capacity, while caring about future generations. For that purpose, we build an original cooperative game framework, where countries can form coalitions in order to optimize their discounted consumption stream in the long-run, within the limits of their stock of natural resources. We use the concept of the recursive core that satisfies both coalitional stability and time consistency. We show that this set is nonempty, stating that an international long-term agreement along the optimal path will be self-enforcing. The presented model can be viewed as a tool to refresh the common look at the North-South opposition and sets the conceptual framework for the exploration of a fair sharing of the fruits of global economic growth.
\end{abstract}

\section{Keywords:}

Non-renewable natural resources, Cooperative games, Recursive core

\section{JEL codes:}

C71, C61, F42, Q20, Q32, Q56

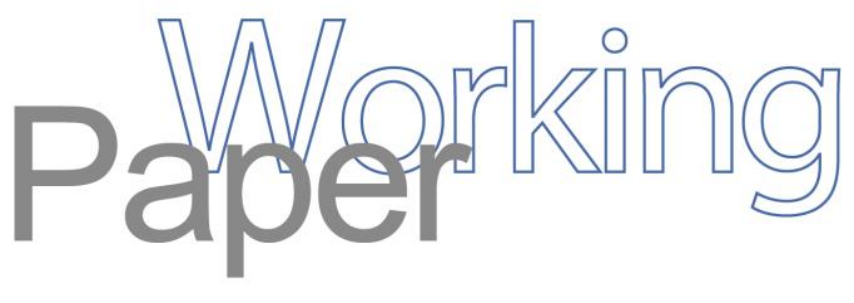




\title{
Sharing the Global Benefits of Finite Natural Resource Exploitation: A Dynamic Coalitional Stability Perspective
}

\author{
Stéphane GONZALEZ* and Fatma Zahra ROSTOM ${ }^{\dagger}$ \\ e-mails: stephane.gonzalez@univ-st-etienne.fr,fatma.rostom@univ-paris1.fr
}

Version of December 19, 2019

\begin{abstract}
The article explores the implications of natural resource scarcity in terms of global cooperation and trade. We investigate whether there exist stable international long-term agreements that take into account the disparities between countries in terms of geological endowments and productive capacity, while caring about future generations. For that purpose, we build an original cooperative game framework, where countries can form coalitions in order to optimize their discounted consumption stream in the long-run, within the limits of their stock of natural resources. We use the concept of the recursive core that satisfies both coalitional stability and time consistency. We show that this set is nonempty, stating that an international long-term agreement along the optimal path will be self-enforcing. The presented model can be viewed as a tool to refresh the common look at the North-South opposition and sets the conceptual framework for the exploration of a fair sharing of the fruits of global economic growth.
\end{abstract}

Keywords: Non-renewable natural resources, Cooperative games, Recursive core

\section{Introduction}

The nexus between trade and mineral resource extraction is crucial to the understanding of wealth creation dynamics. Early stages of mankind are named after the materials from which tools and weapons were made of: the Bronze Age, which arose fully around 3000 BCE, was preceded by the Chalcolithic or Copper Age. The Near East was the "kernel of the Age of Metals", but was poorly endowed with these materials. Therefore, the "valley urban societies" had to exchange with the "barbarians" (in the technical archaeological sense), before trade expanded from the Near East to Europe (Goody, 2012). Modern resource extraction and trade are of much higher orders of magnitude. Industrialization is characterized by the construction of infrastructures in the sectors of heavy industry, energy, housing, transport and communication, and therefore is inevitably associated with an increase in the consumption of raw materials. The development of trade, necessary to meet an ever-growing demand, is directly linked to the institutional implementation of free trade agreements in free trade areas.

The debate on the "trade-environment divide" (Esty, 2001) is mostly focused on pollution issues (Copeland and Taylor, 1994). The interaction between trade and materially sustainable

\footnotetext{
*Univ Lyon, UJM Saint-Etienne, GATE UMR 5824, F-42023 Saint- Etienne

${ }^{\dagger}$ Université Paris 1, Centre d'Économie de la Sorbonne, Chaire Énergie et Prospérité
} 
growth (Dupuy, 2014) was studied by Asheim (1986) who looked at the effects of opening economies on the Hartwick rule. Other models focus on two-region trade, the industrialized North versus the resource exporter South (Chichilnisky, 1993). A rich literature studies the socalled "resource curse" (Gaitan and Roe, 2012) or the formation of cartels (Kemp and Van Long, 1984) through the traditional Heckscher and Ohlin model (Heckscher and Ohlin, 1991). Strategic game theory approaches were also used to explore finite resources issues (Van Long, 2011), and the relationship with trade was studied with two countries (Kagan et al., 2015; Tamasiga and Bondarev, 2014). However, and despite the call of some game theorists to develop cooperative game theory (Samuelson, 2016), the literature on material sustainability neglected to treat the issue through cooperative lenses. Trade can indeed be analyzed through three concepts: "pure competition", "coalitional power" and "fair division". Shapley and Shubik (1969) provide a static market game for the three varieties of solution and show that, under certain assumptions, the outcomes converge. In a distinct stream of literature of cooperative games, the question of renewable and common-pool resources was studied (Funaki and Yamato, 1999), mostly for fishery or river sharing (Ambec and Sprumont, 2002; Béal et al., 2013) problems. However, these models do not take into account the dynamic aspects of the issue.

The aim of this article is to show that there exist stable international long-term agreements that take into account the disparities between countries in terms of geological endowments and productive capacity, while caring about future generations. In this context, countries can form coalitions and look for the best agreement to optimize their allocation. Members can break an alliance according to their interest, at any point of time. Dynamic cooperative game theory is the most fitted framework to tackle this kind of issues, but the literature on the application of such games is quite limited. Some dynamic cooperative approaches were used to tackle environmental issues, especially fisheries (Munro, 1979) and pollution (Jørgensen and Zaccour, 2001; Hoofe, 2019). The question of non-renewable resource exhaustibility was raised by d'Albis and Ambec (2010), who study cooperative allocations among overlapping generations depleting a natural resource over an infinite future. However, the authors focus on intergenerational allocations and do not include any international aspect in their model.

In order to find such long-term international agreements, the twin issues of coalitional stability and time consistency have to be handled at once. First, it is a question of designing a policy that no coalition of the present generation has an incentive to refuse. Traditionally, this issue is tackled by the concept of the core, which is the set of allocations which cannot be improved upon by any coalition of agents. However, this notion does not deal with the dynamic issues relevant to the intergenerational sharing of the benefits generated from resource extraction. It must therefore also ensure that at no point in time the policy initiated then departs from the one originally planned. Recent papers tackle the issue of dynamic core concepts (Predtetchinski et al., 2004; Kranich et al., 2005; Lehrer and Scarsini, 2013), but the first studies date back to the 70's with the introduction of core concepts for production economies (Boehm, 1974; Becker, 1982), as well as the notion of trust in a monetary economy (Gale, 1978). In the present article, we use the solution concept of the recursive core, defined by Becker and Chakrabarti (1995) as a set of allocations for which no coalition can improve upon its consumption stream at any time given its accumulation of assets up to that period. This concept satisfies both properties of coalitional stability and time consistency.

The novelty of our approach is to model the geographical heterogeneity of natural endowments that results from geological processes, as well as the geographical heterogeneity of capital and technological endowments that results from historical processes. For that purpose, we settle a cooperative game where countries can form coalitions in order to optimize their discounted consumption stream in the long-run, within the limits of their stock of natural resources. Trade is viewed as a market cooperative TU game à la Shapley and Shubik (1969) where non-renewable 
natural resource inputs are exchanged in such a way as to maximize the total value to be shared among the coalition. This game is cast into a traditional Ramsey-type model of intergenerational equity, where the Bellman's dynamic programming framework is used. An international long-term policy is translated into:

- An action strategy taken by a coalition $S$. It consists in a quota of natural resources extraction, a commercial quota and an investment plan proposed to each country at each time $t$. An action strategy is optimal for $S$ if the commercial quota optimizes the current collective production of $S$, and if the extraction quota and the investment plan ensure the maximization of long-term consumption.

- A distribution policy of collectively produced consumption goods. Such a policy is said to be efficient and coalitionally rational if, from some date $t$ onward, a coalition cannot block an international agreement by undertaking a policy increasing its own long-term consumption.

In other words, the coalition decides its extraction and investment plan, and deduces the amount of resources it wishes to trade. It then distributes consumption goods to its members. We show that there exists a unique optimal path of extraction and investment for each country in a coalition $S$, which could be interpreted as quotas proposed by a social planner. As traded quantities are also unique, we conclude that there exists a unique optimal path for collective production in a coalition $S$. We then can build an intertemporal cooperative game where the worth of $S$ is the discounted sum of consumption - corresponding to the remaining part of production that is not invested - along this optimal path. We demonstrate that the recursive core associated to this game is nonempty. This result states that an international long-term agreement along the optimal path will be self-enforcing. The countries, as rational agents, will stick to this agreement, as no other coalition could offer them a better outcome, at any point in time. Furthermore, we show that this recursive core contains an infinity of elements, opening an avenue for the research of a fair distribution from both intergenerational and international points of view.

The article is organized as follows. The following section presents our formal apparatus and details the reasoning of the model. In the third section, our main results and their interpretation are provided. The last section is a discussion on the model and the research avenue it opens. All proofs are available in the appendix.

\section{A Formal Statement of the Problem}

\section{$2.1 \quad$ Game Set-Up}

Let $N$ be a fixed and finite nonempty set of countries, who are the game players. Countries have the possibility to form a coalition which can be viewed as a free-trade area. Each country $i \in N$ has a natural resource ${ }^{1}$ that can be exploited by the infinitely lived country at each period $t \in \mathbb{N}$. Growth is constrained by natural resource availability, its costs of extraction, as well as the accumulation of capital. Countries have three decisions to make at each period: resource extraction, resource trade and investment level through savings that will increase the production capacity of the next period. The remaining created flow of wealth is consumed, and generations seek to maximize their present consumption as well as the discounted consumption of their successors. The present model is therefore a cooperative games adaptation of Ramsey's benchmark model with traded mineral resources.

\footnotetext{
${ }^{1}$ Note that this model could be extended to a finite number of resources.
} 
At period $t$ and for a nonempty coalition $S \subseteq N$, consider an amount $\breve{y}_{t}(S)$ of a single final good, which can be viewed as Ricardo's metaphorical corn in neoclassical theory. The coalition chooses the amount $\eta_{i, t}$ each country $i$ will invest for tomorrow (the seeds to be sowed next year) to increase the country's stock of capital $K_{i, t}$. This leaves $\breve{y}_{t}(S)-\sum_{i \in S} \eta_{i, t}$ to the present consumption of the coalition. The dynamics of $i$ 's capital stock is given by the following law of motion, $\tau$ being the depreciation rate:

$$
K_{i, t+1}=K_{i, t}+\eta_{i, t}-\tau K_{i, t}
$$

The coalition also chooses the quantity $e_{i, t}$ of resources each country will extract from its available stock $\Omega_{i, t}$. The dynamics of $i$ 's resource stock is given by the following law of motion:

$$
\Omega_{i, t+1}=\Omega_{i, t}-e_{i, t}
$$

Extracted resources are exchanged in a free-trade area with no transaction cost, and country $i$ ends up with a quantity $z_{i, t}$ of input for its domestic production. The country's productive sector then uses its production capacity $K_{i, t}$ and $z_{i, t}$ to produce $f_{i}\left(z_{i}, K_{i}\right)$ through a raw countryspecific production function $f_{i}$. We assume that, for each $i \in S, f_{i}: \mathbb{R}_{+} \times \mathbb{R}_{+} \rightarrow \mathbb{R}_{+}$satisfies:

(i) $f_{i}$ is continuous, non-decreasing, strictly concave;

(ii) $f_{i}\left(z_{i}, 0\right)=0$ for each $z_{i} \in \mathbb{R}_{+}$;

(iii) $f_{i}$ is differentiable with respect to $K_{i}$;

(iv) $\lim _{K_{i} \rightarrow 0} \frac{\partial f_{i}\left(z_{i}, K_{i}\right)}{\partial K_{i}}>\tau$ for each $z_{i} \in \mathbb{R}_{+}$;

(v) $\lim _{K_{i} \rightarrow \infty} \frac{\partial f_{i}\left(z_{i}, K_{i}\right)}{\partial K_{i}}<\tau$ for each $z_{i} \in \mathbb{R}_{+}$.

Note that these conditions are weaker than the Inada conditions. The cost of extraction $c_{i}\left(e_{i, t}, \Omega_{i, t}\right)$ of $i \in N$, expressed in consumption units, is represented through a continuous convex function depending both on the quantity $\Omega_{i, t}$ available to the country $i$ and the quantity $e_{i, t} \in\left[0, \Omega_{i, t}\right]$ extracted at date $t$.

We consider that each country $i$ has an initial endowment $\breve{y}_{i, 0}$ and that

$$
\breve{y}_{0}(S)=\sum_{i \in S} \breve{y}_{i, 0} \cdot
$$

The production process takes one period to be achieved, since time is necessary for natural resource transportation and capital usage. The choice of the trading strategy is made such that the total net production $\breve{y}_{t+1}(S)$ of each coalition $S$ is maximized through the following static optimization problem:

$$
\underset{\left(z_{i, t}\right)_{i \in S}}{\operatorname{maximize}} \sum_{i \in S} f_{i}\left(z_{i, t}, K_{i, t}\right)-c_{i}\left(e_{i, t}, \Omega_{i, t}\right)
$$

subject to

$$
\sum_{i \in S} z_{i, t}=\sum_{i \in S} e_{i, t}
$$

Fig. 2.1 presents a scheme of the model at $t$ and $t+1$. 


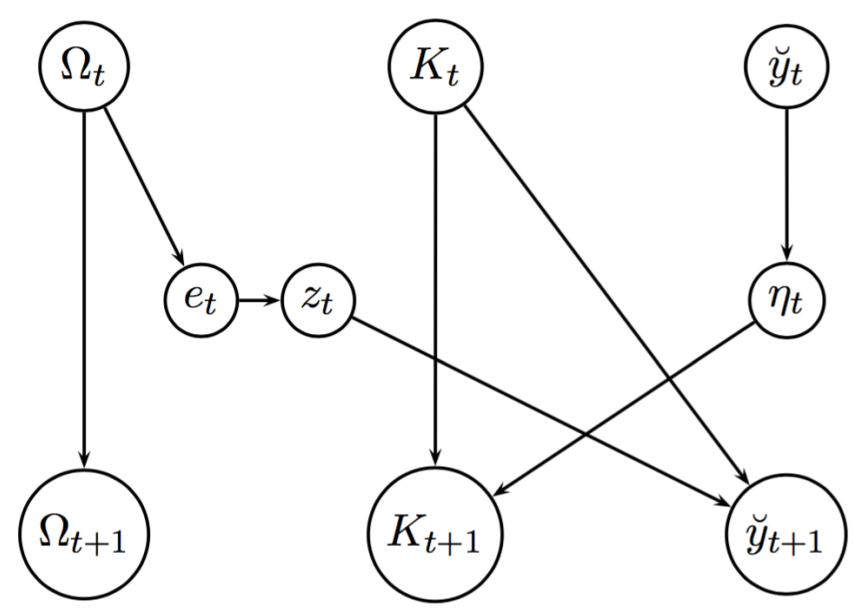

Figure 1: Scheme of the state and action dynamics

\subsection{Significant Sets}

We define $\bar{\Omega} \in \mathbb{R}_{+}$as the total and finite amount of resources on Earth. Following neoclassical arguments, the assumptions on the function $f_{i}$ as well as the dynamics of $K_{i}$ ensure that there exists $\bar{K} \in \mathbb{R}_{+}$such that for each $i \in S$ and for each $t \in \mathbb{N}, K_{i, t} \leq \bar{K}$. As a consequence, there exists $\overline{\breve{y}} \in \mathbb{R}_{+}$such that for each $t \in \mathbb{N}, \breve{y}_{t}(S) \leq \overline{\breve{y}}$.

Let $\Omega(S)=\left(\Omega_{i}\right)_{i \in S}$ (or simply $\Omega$ if there is no danger of confusion) be the vector of natural resource stocks and $K(S)=\left(K_{i}\right)_{i \in S}$ (or simply $K$ if there is no danger of confusion) be the vector of capital stocks. The state space $\mathcal{S}(S)$ is defined by:

$$
\mathcal{S}(S):=\left\{(\Omega(S), K(S), \breve{y}(S)) \in \mathbb{R}_{+}^{S} \times \mathbb{R}_{+}^{S} \times \mathbb{R}_{+}, \breve{y}(S) \leq \bar{y} \text { and } \forall i \in S, \Omega_{i} \leq \bar{\Omega}, K_{i} \leq \bar{K}\right\} .
$$

Let $e(S)=\left(e_{i}\right)_{i \in S}$ (or simply $e$ if there is no danger of confusion) be the vector of extraction and $\eta(S)=\left(\eta_{i}\right)_{i \in S}$ (or simply $\eta$ if there is no danger of confusion) be the vector of investment. The action space $\mathcal{A}(S)$ is defined by:

$$
\mathcal{A}(S):=\left\{(e(S), \eta(S)) \in \mathbb{R}_{+}^{S} \times \mathbb{R}_{+}^{S}, \forall i \in S, e_{i} \leq \bar{\Omega}, \eta_{i} \leq \overline{\breve{y}}\right\} .
$$

The set of feasible vectors of extraction $e(S)$ for a coalition $S$ with a stock described by $\Omega(S)$ is

$$
E^{\Omega}(S):=\left\{e(S) \in\left(\mathbb{R}_{+}\right)^{S}, e_{i} \leq \Omega_{i}\right\} .
$$

We now define the set of feasible vectors of investment $\eta(S)$ for a coalition $S$ by

$$
\mathcal{I}^{\breve{y}(S)}(S):=\left\{\eta(S) \in\left(\mathbb{R}_{+}\right)^{S}, \sum_{i \in S} \eta_{i} \leq \breve{y}(S)\right\} .
$$

We can therefore define the set of couples $(e(S), \eta(S))$ of feasible actions by:

$$
\Phi^{\Omega, \breve{y}(S)}(S):=\left\{(e(S), \eta(S)) \mid e(S) \in E^{\Omega}(S), \eta(S) \in \mathcal{I}^{\breve{y}(S)}(S)\right\},
$$

Now, the set of available resource input after the exchange $z(S)=\left(z_{i}\right)_{i \in S}$ (or simply $z$ if there is no danger of confusion) is:

$$
Z^{e}(S):=\left\{z(S) \in\left(\mathbb{R}_{+}\right)^{S}, \sum_{i \in S} z_{i}=\sum_{i \in S} e_{i}\right\}
$$




\subsection{Coalitional and Intergenerational Worth}

At each period, countries belonging to a coalition $S$ when the production is $\breve{y}(S)$ and the investment is $\eta \in \mathcal{I}^{\breve{y}(S)}(S)$ will earn a worth $v^{\breve{y}(S), \eta}(S)$ that shall not be more than their consumption:

$$
\forall \breve{y}(S) \in \mathbb{R}_{+}, \forall \eta \in \mathcal{I}^{\breve{y}(S)}(S) v^{\breve{y}(S), \eta}(S)=\breve{y}(S)-\sum_{i \in S} \eta_{i} .
$$

Let us now zoom up in the time scale, and describe the behavior of countries from an intertemporal viewpoint. Let $\delta \in] 0,1\left[\right.$ be the discount factor. The intertemporal worth $V^{\Omega_{0}, K_{0}, \breve{y}_{0}(S)}(S)$ of the coalition $S$ is defined as the value of the following dynamic programming problem:

$$
\begin{array}{cl}
\underset{\left(e_{t}, \eta_{t}\right)_{t \in \mathbb{N}}}{\operatorname{maximize}} & \sum_{t \in \mathbb{N}} \delta^{t} v^{\breve{y}_{t}(S), \eta_{t}}(S) \\
\text { subject to } & \\
& \Omega_{t+1}=\Omega_{t}-e_{t}, \quad t \in \mathbb{N} \\
& K_{t+1}=(1-\tau) K_{t}+\eta_{t}, \quad t \in \mathbb{N} \\
& \breve{y}_{t+1}(S)=\max \left\{\sum_{i \in S} f_{i}\left(z_{i, t}, K_{i, t}\right)-c_{i}\left(e_{i, t}, \Omega_{i, t}\right),\left(z_{i, t}\right)_{i \in S} \in Z^{e}(S)\right\}, \quad t \in \mathbb{N} \\
& \left(e_{t}, \eta_{t}\right) \in \Phi^{\Omega_{t}, \breve{y}_{t}(S)}(S), t \in \mathbb{N}
\end{array}
$$

\section{Existence of Distribution Policies Along the Optimal Path}

\subsection{Optimal Action Strategy}

The following proposition establishes that, given a flow of resource extracted by each country, there exists a unique optimal way to trade that can be interpreted as an optimal commercial quota.

Proposition 1. The function $(\Omega, e, K) \mapsto \max \left\{\sum_{i \in S} f_{i}\left(z_{i}, K_{i}\right)-c_{i}\left(e_{i}, \Omega_{i}\right),\left(z_{i}\right)_{i \in S} \in Z^{e}(S)\right\}$ is continuous and strictly concave over $[0, \bar{\Omega}]^{S} \times[0, \bar{\Omega}]^{S} \times[0, \bar{K}]^{S}$. Furthermore, for each $(\Omega, e, K) \in$ $[0, \bar{\Omega}]^{S} \times[0, \bar{\Omega}]^{S} \times[0, \bar{K}]^{S}$, there exists a unique $z^{*} \in Z^{e}(S)$ - continuously varying with $(\Omega, e, K)$ - such that

$$
\max \left\{\sum_{i \in S} f_{i}\left(z_{i}, K_{i}\right)-c_{i}\left(e_{i}, \Omega_{i}\right),\left(z_{i}\right)_{i \in S} \in Z^{e}(S)\right\}=\sum_{i \in S} f_{i}\left(z_{i}^{*}, K_{i}\right)-c_{i}\left(e_{i}, \Omega_{i}\right) .
$$

The next proposition states that, given the initial endowments of each country, there exists a unique optimal way to extract and to invest that can be interpreted as optimal extraction quota and investment plan.

Proposition 2. For each nonempty coalition $S \subseteq N$, and each $\left(\Omega_{i, 0}\right)_{i \in S} \in[0, \bar{\Omega}]^{S},\left(K_{i, 0}\right)_{i \in S} \in$ $[0, \bar{K}]^{S}$ and $\breve{y}_{0}(S) \in[0, \bar{y}]$, there exists a unique optimal path of extraction and investment to the dynamic programming problem (1).

\subsection{Efficient and Coalitionally Rational Distribution Policy}

We showed that there exists a unique optimal path of extraction and investment for each country in a coalition $S$, which could be interpreted as quotas imposed by a social planner. This leads to a unique path of resource and capital stocks. As traded quantities are also unique, we 
can conclude that there exists a unique path for collective production in a coalition $S$. The consumption path of each country, for its part, is not unique and depends upon the sharing rule decided by the coalition. The following result states that an international long-term agreement along this optimal path will be self-enforcing. The countries, as rational agents, will stick to this agreement, as no other coalition could offer them a better outcome, at any point in time. In other words, this agreement within $N$ cannot be dominated by any coalition $S \subseteq N$. For that purpose, we adapt the concept of recursive core to our framework. This concept satisfies the properties of efficiency, coalitional rationality and also tackles the crucial issue of time consistency. Let us now formally introduce this concept.

For each initial state $(\Omega, K, \breve{y}) \in[0 ; \bar{\Omega}]^{N} \times[0, \bar{K}]^{N} \times[0, \bar{y}]$ of the grand coalition $N$, there exists a unique optimal extraction and investment solution $\left(e_{t}^{*}(N), \eta_{t}^{*}(N)\right)_{t \in \mathbb{N}}$ to the dynamic programming problem (1) according to Proposition 2. Let $\left(\Omega_{t}^{*}(N), K_{t}^{*}(N), \breve{y}_{t}^{*}(N)\right)_{t \in \mathbb{N}}$ be the state evolution corresponding to the optimal path when the initial state is $(\Omega, K, \breve{y})$. Denote by $\left(\Omega_{S, t}^{*}(N), K_{S, t}^{*}(N), \breve{y}_{S, t}^{*}(N)\right)$ the projection $\left(\Omega_{i, t}^{*}(N), K_{i, t}^{*}(N), \breve{y}_{i, t}^{*}(N)\right)_{i \in S}$ of $\left(\Omega_{t}^{*}(N), K_{t}^{*}(N), \breve{y}_{t}^{*}(N)\right)$. Note that the consumption path, for its part, is not unique. An international distribution policy, $\pi$, is a mapping which associates with each initial state $(\Omega, K, \breve{y})$ a path of consumption vector $\left(\pi_{i, t}(\Omega, K, \breve{y})\right)_{i \in N, t \in \mathbb{N}}$ such that $\forall t \in \mathbb{N}$ :

$$
\sum_{i \in N} \pi_{i, t}(\Omega, K, \breve{y})=\breve{y}_{t}^{*}(N)-\sum_{i \in N} \eta_{i, t}^{*}(N)
$$

A policy $\pi$ is dominated by $S$ at date $T$ if there exists an initial state $(\Omega, K, \breve{y})$ such that:

$$
\sum_{t=0}^{+\infty} \delta^{t} \sum_{i \in S} \pi_{i, t+T}(\Omega, K, \breve{y})<V^{\Omega_{S, T}^{*}(N), K_{S, T}^{*}(N), \breve{y}_{S, T}^{*}(N)}(S) .
$$

The recursive core $\mathcal{C}(\Omega, K, \breve{y})$ is the set of undominated policies when the initial state is $(\Omega, K, \breve{y})$. The following theorem states that the recursive core of this set-up is nonempty whatever the initial state is. It further indicates that this core contains an infinity of elements, corresponding to an infinite way of distributing consumption streams among the countries of the international coalition.

Theorem 1. For each $(\Omega, K, \breve{y}) \in[0 ; \bar{\Omega}]^{N} \times[0, \bar{K}]^{N} \times[0, \bar{y}], \mathcal{C}(\Omega, K, \breve{y})$ contains an infinity of elements.

This theorem states that, if the countries of a coalition follow a long-term cooperation strategy in terms of extraction, investment and induced trade, then the best way to optimize their own consumption while caring about future generations is to form a global coalition. The recursive core is constituted of the streams of consumption to be allocated to each country. Since this core is not reduced to a singleton, it is possible to compare the different distribution policies and to select a policy among these allocations. For instance, a fairness criteria could be developed in the tradition of the axiomatic methodology in order to explore possible ethic consumption paths. This last point, however, is beyond the scope of this paper and constitutes an open avenue for further research. An interesting lead would be to study the nucleolus solution concept (Schmeidler, 1969), which maximizes recursively the 'welfare' of the worst treated coalitions and can be understood as an application of the Rawlsian social welfare function (Hamlen et al., 1977). Another lead would be to study a time-consistent Shapley value (Petrosjan and Zaccour, 2003) and to look whether it belongs to the recursive core in our particular setting.

Note that the instantaneous core - the core of $v^{\breve{y}_{t}, \eta_{t}}$ at each period $t$-can be empty even if the overall core - the core of $V^{\Omega_{0}, K_{0}, \breve{y}_{0}}$ - is not (see Lehrer and Scarsini (2013) for a discussion on this point). A more profound analysis of the short-run efficient and coalitionaly rational policies could also be the subject of further research. 


\section{Concluding Remarks}

We showed that an international cooperation strategy leads to a unique optimal path of collective production since all the processes involved in this production - namely the extraction of natural resources from a finite stock, their trade and the evolution of the productive capacity - should be unique to be optimal. On the demand side, a given part of this production is optimally allocated to the investment needed to build the productive capacity of each country, in a unique manner. The remaining part of production, corresponding to the consumption of goods, can be shared among countries, this time in different manners. The optimal paths could be interpreted as resulting from quotas chosen by a benevolent planner, but this interpretation that we propose could be objected. Indeed, imposing commercial quotas is not consistent with a common definition of free trade. This objection is fully anchored in the socialist calculation debate (O'Neill, 2004; Stiglitz, 1996; Bardhan and Roemer, 1992) and is not the subject of this study. Note however that, by definition of optimality, agents should not deviate from these quotas even in a free market interpretation. Another alternative interpretation would be the result of a negotiation procedure between countries. In all cases, no country would have the incentive to refuse a core allocation. This last interpretation is linked to the question of the implementation of cooperative game solutions, also called "Nash program". This could be the subject of a subsequent paper.

This set-up provides a basis on which to progress on questions related to the long-term material development of countries. We support the idea that cooperative game theory provides a fresh and fairly simple framework to tackle traditional issues raised in growth theory, since it better takes into account the heterogeneity of a finite number of countries. An interesting question would be to characterize technological catch-up effects between countries and how it affects the balance of power, by adding a time-dependent production function or an endogenous knowledge capital accumulation. Moreover, recycling or pollution effects could be investigated in our framework and lead to a more holistic understanding of the sustainability of our "Spaceship Earth" (Boulding, 1966).

\section{Acknowledgments}

This work was first developed as a chapter of Fatma Zahra Rostom's PhD dissertation. We thank the $\mathrm{PhD}$ referees for their helpful comments and suggestions. This paper was presented at the 19th Annual SAET Conference and the International Conference of Public Economic Theory 2019. Financial support of IDEXLYON from Université de Lyon (project INDEPTH) within the Programme Investissements d'Avenir (ANR-16-IDEX-0005) and the Chaire "Énergie et Prospérité" are gratefully acknowledged.

\section{References}

Stefan Ambec and Yves Sprumont. Sharing a river. Journal of Economic Theory, 107:453-462, 2002.

Geir B Asheim. Hartwick's rule in open economies. Canadian Journal of Economics, pages 395-402, 1986.

Pranab Bardhan and John E Roemer. Market socialism: a case for rejuvenation. Journal of Economic Perspectives, 6(3):101-116, 1992. 
Sylvain Béal, Amandine Ghintran, Éric Rémila, and Philippe Solal. The river sharing problem: A survey. International Game Theory Review, 15(03):1340016, 2013.

Robert A Becker. The existence of core allocations in a one sector model of capital accumulation. Economics Letters, 9(3):201-207, 1982.

Robert A Becker and Subir K Chakrabarti. The recursive core. Econometrica: Journal of the Econometric Society, pages 401-423, 1995.

Volker Boehm. The core of an economy with production. The Review of Economic Studies, 41 (3):429-436, 1974.

Olga Bondareva. Some applications of linear programming methods to the theory of cooperative games. Problemy Kibernetiki, 10:119-139, 1963.

Kenneth E Boulding. The economics of the coming spaceship earth. In Sixth Resources for the Future Forum on Environmental Quality in a Growing Economy, 1966.

Graciela Chichilnisky. North-south trade and the dynamics of renewable resources. Structural Change and Economic Dynamics, 4(2):219-248, 1993.

Brian R Copeland and M Scott Taylor. North-south trade and the environment. The quarterly journal of Economics, 109(3):755-787, 1994.

Hippolyte d'Albis and Stefan Ambec. Fair intergenerational sharing of a natural resource. Mathematical Social Sciences, 59(2):170-183, 2010.

Louis Dupuy. Sustainability and International Trade. PhD thesis, Université de Bordeaux, 2014.

Daniel C Esty. Bridging the trade-environment divide. Journal of Economic Perspectives, 15 (3):113-130, 2001.

Yukihiko Funaki and Takehiko Yamato. The core of an economy with a common pool resource: A partition function form approach. International Journal of Game Theory, 28(2):157-171, 1999.

Beatriz Gaitan and Terry L Roe. International trade, exhaustible-resource abundance and economic growth. Review of Economic Dynamics, 15(1):72-93, 2012.

Douglas Gale. The core of a monetary economy without trust. Journal of Economic Theory, 19(2):456-491, 1978.

Stéphane Gonzalez and Michel Grabisch. Autonomous coalitions. Annals of Operations Research, 235(1):301-317, 2015.

Jack Goody. Metals, culture and capitalism: an essay on the origins of the modern world. Cambridge University Press, 2012.

Susan S Hamlen, William A Hamlen, and John T Tschirhart. The use of core theory in evaluating joint cost allocation schemes. The Accounting Review, 52(3):616, 1977.

Eli Filip Heckscher and Bertil Gotthard Ohlin. Heckscher-Ohlin trade theory. The MIT Press, 1991.

Simon Hoofe. Linear-state differential games in partition function form. International Game Theory Review, 2019. 
Steffen Jørgensen and Georges Zaccour. Time consistent side payments in a dynamic game of downstream pollution. Journal of economic dynamics and control, 25(12):1973-1987, 2001.

Mark Kagan, Frederick Van der Ploeg, and Cees Withagen. Battle for climate and scarcity rents: beyond the linear-quadratic case. Dynamic games and applications, 5(4):493-522, 2015.

Murray C Kemp and Ngo Van Long. The role of natural resources in trade models. Handbook of international economics, 1:367-417, 1984.

Laurence Kranich, Andrés Perea, and Hans Peters. Core concepts for dynamic tu games. International Game Theory Review, 7(01):43-61, 2005.

Ehud Lehrer and Marco Scarsini. On the core of dynamic cooperative games. Dynamic Games and Applications, 3(3):359-373, 2013.

Gordon R Munro. The optimal management of transboundary renewable resources. Economic Studies, 41:37-50, 1979.

John O'Neill. Ecological economics and the politics of knowledge: the debate between hayek and neurath. Cambridge Journal of Economics, 28(3):431-447, 2004.

Leon Petrosjan and Georges Zaccour. Time-consistent shapley value allocation of pollution cost reduction. Journal of economic dynamics and control, 27(3):381-398, 2003.

Arkadi Predtetchinski, Jean-Jacques Herings, and Hans Peters. The strong sequential core in a dynamic exchange economy. Economic Theory, 24(1):147-162, 2004.

Larry Samuelson. Game theory in economics and beyond. Journal of Economic Perspectives, 30(4):107-30, 2016.

David Schmeidler. The nucleolus of a characteristic function game. SIAM Journal on applied mathematics, 17(6):1163-1170, 1969.

Lloyd S Shapley. On balanced sets and cores. Naval Research Logistics Quaterly, 14:453-460, 1967.

Lloyd S Shapley and Martin Shubik. Pure competition, coalitional power, and fair division. International Economic Review, 10(3):337-362, 1969.

Joseph E Stiglitz. Whither socialism? MIT press, 1996.

Rangarajan K Sundaram. A first course in optimization theory. Cambridge university press, 1996.

Phemelo Tamasiga and Anton Bondarev. Differential games approach to trade with exhaustible resources, 2014.

Ngo Van Long. Dynamic games in the economics of natural resources: a survey. Dynamic Games and Applications, 1(1):115-148, 2011. 


\section{Appendix A}

\section{Proof of Proposition 1}

Proof. Let $g:[0, \bar{\Omega}]^{S} \times[0, \bar{\Omega}]^{S} \times[0, \bar{K}]^{S} \times \mathbb{R}_{+}^{S} \rightarrow \mathbb{R}_{+}$be the function defined as

$$
g((\Omega, e, K), z)=\sum_{i \in S}\left(f_{i}\left(z_{i}, K_{i}\right)-c_{i}\left(e_{i}, \Omega_{i}\right)\right),
$$

and $C:[0, \bar{\Omega}]^{S} \times[0, \bar{\Omega}]^{S} \times[0, \bar{K}]^{S} \rightrightarrows \mathbb{R}_{+}^{S}$ be the correspondence defined as

$$
C(\Omega, e, K)=Z^{e}(S) .
$$

By continuity and strict concavity of $f_{i}$ and $-c_{i}$ for each $i \in S$, function $g$ is continuous and strictly concave on $[0, \bar{\Omega}]^{S} \times[0, \bar{\Omega}]^{S} \times[0, \bar{K}]^{S} \times \mathbb{R}_{+}^{S}$. Clearly, $C$ is a compact-valued continuous correspondence on $[0, \bar{\Omega}]^{S} \times[0, \bar{\Omega}]^{S} \times[0, \bar{K}]^{S}$ and has a convex graph. We have the needed conditions to apply Berge's Maximum Theorem under Convexity (Sundaram, 1996, p.237). We conclude that $\max \{g((\Omega, e, K), z), z \in C(\Omega, e, K)\}$ is a continuous and strictly concave function on $[0, \bar{\Omega}]^{S} \times[0, \bar{\Omega}]^{S} \times[0, \bar{K}]^{S}$ and $C^{*}(\Omega, e, K)=\operatorname{argmax}\{g((\Omega, e, K), z), z \in C(\Omega, e, K)\}$ is a continuous single-valued function.

\section{Proof of Proposition 2}

Proof. Existence. The existence of an optimal path of extraction and investment is a direct application of a well-established theorem of existence in dynamic optimization (see for instance Theorem 12.19 in Sundaram (1996, p.298)). Indeed,

(i) The reward function $(\breve{y}(S), \eta) \mapsto v^{\breve{y}(S), \eta}(S)$ is continuous and bounded on $[0, \bar{y}] \times[0, \bar{y}]^{S}$.

(ii) It is clear that $(\Omega, e) \mapsto \Omega-e$ is continuous from $[0, \bar{\Omega}]^{S} \times[0, \bar{\Omega}]^{S}$ to $[0, \bar{\Omega}]^{S}$. In addition, $(K, \eta) \mapsto(1-\tau) K+\eta$ is continuous from $[0, \bar{K}]^{S} \times[0, \bar{y}]^{S}$ to $[0, \bar{K}]^{S}$. By Proposition 1 , $(\Omega, e, K) \mapsto \max \left\{\sum_{i \in S} f_{i}\left(z_{i}, K_{i}\right)-c_{i}\left(e_{i}, \Omega_{i}\right),\left(z_{i}\right)_{i \in S} \in Z^{e}(S)\right\}$ is continuous on $[0, \bar{\Omega}]^{S} \times$ $[0, \bar{\Omega}]^{S} \times[0, \bar{K}]^{S}$. Hence, the transition function is continuous.

(iii) The feasible action correspondence $(\Omega, \breve{y}(S)) \mapsto \Phi^{\Omega, \breve{y}(S)}(S)$ is compact-valued and continuous.

Uniqueness. Assume by way of contradiction that there exist two different optimal paths $\left(e_{t}^{0}, \eta_{t}^{0}\right)_{t \in \mathbb{N}}$ and $\left(e_{t}^{1}, \eta_{t}^{1}\right)_{t \in \mathbb{N}}$ when the initial vector is $\left(\Omega_{0}, K_{0}, \breve{y}_{0}(S)\right)$ for a coalition $S$.

We denote by $\left(\Omega_{t}^{0}\right)_{t \in \mathbb{N}}$ and $\left(\Omega_{t}^{1}\right)_{t \in \mathbb{N}}$ the respective sequence of resource stock vectors defined by $\Omega_{0}^{0}=\Omega_{0}, \Omega_{0}^{1}=\Omega_{0}$ and for each $t>0, \Omega_{t+1}^{0}=\Omega_{t}^{0}-e_{t}^{0}$ and $\Omega_{t+1}^{1}=\Omega_{t}^{1}-e_{t}^{1}$. We denote by $\left(K_{t}^{0}\right)_{t \in \mathbb{N}}$ and $\left(K_{t}^{1}\right)_{t \in \mathbb{N}}$ the respective sequence of capital stock vectors defined by $K_{0}^{0}=K_{0}$, $K_{0}^{1}=K_{0}$ and for each $t>0, K_{t+1}^{0}=(1-\tau) K_{t}^{0}+\eta_{t}^{0}$ and $K_{t+1}^{1}=(1-\tau) K_{t}^{1}+\eta_{t}^{1}$. For each $\alpha \in[0,1]$, we define the path of extraction $\left(e_{t}^{\alpha}\right)_{t \in \mathbb{N}}$ as follows:

$$
\forall t \in \mathbb{N}, e_{t}^{\alpha}=\alpha e_{t}^{1}+(1-\alpha) e_{t}^{0}
$$

the path of investment $\left(\eta_{t}^{\alpha}\right)_{t \in \mathbb{N}}$ is defined as:

$$
\forall t \in \mathbb{N}, \eta_{t}^{\alpha}=\alpha \eta_{t}^{1}+(1-\alpha) \eta_{t}^{0} .
$$

Let $\left(\Omega_{t}^{\alpha}\right)_{t \in \mathbb{N}}$ be the sequence of resource stock vectors defined by $\Omega_{0}^{\alpha}=\Omega_{0}$ and for each $t \in \mathbb{N}$ by $\Omega_{t+1}^{\alpha}=\Omega_{t}^{\alpha}-e_{t}^{\alpha}$. A simple induction leads to $\Omega_{t}^{\alpha}=\alpha \Omega_{t}^{1}+(1-\alpha) \Omega_{t}^{0}$ for each $t \in \mathbb{N}$. 
Let $\left(K_{t}^{\alpha}\right)_{t \in \mathbb{N}}$ be the sequence of capital stock vectors defined by $K_{0}^{\alpha}=K_{0}$ and for each $t \in \mathbb{N}$ by $K_{t+1}^{\alpha}=(1-\tau) K_{t}^{\alpha}+\eta_{t}^{\alpha}$. A simple induction leads to $K_{t}^{\alpha}=\alpha K_{t}^{1}+(1-\alpha) K_{t}^{0}$ for each $t \in \mathbb{N}$.

We set $\breve{y}_{0}^{\alpha}(S)=\breve{y}_{0}(S)$ and for each $t>0$,

$$
\breve{y}_{t}^{\alpha}(S)=\max \left\{\sum_{i \in S} f_{i}\left(z_{i, t-1}, K_{i, t-1}^{\alpha}\right)-c_{i}\left(e_{i, t-1}^{\alpha}, \Omega_{i, t-1}^{\alpha}\right),\left(z_{i, t-1}\right)_{i \in S} \in Z^{e_{t-1}^{\alpha}}(S)\right\} .
$$

We define for each $t \in \mathbb{N}$ the following vectors ${ }^{2}$ :

$$
\left(z_{i, t}^{* 0}\right)_{i \in S}=\underset{\left(z_{i, t}\right)_{i \in S} \in Z^{e_{t}^{0}(S)}}{\operatorname{argmax}} \sum_{i \in S} f_{i}\left(z_{i, t}, K_{i, t}^{0}\right)-c_{i}\left(e_{i, t}^{0}, \Omega_{i, t}^{0}\right),
$$

and

$$
\left(z_{i, t}^{* 1}\right)_{i \in S}=\underset{\left(z_{i, t}\right)_{i \in S} \in Z^{e_{t}^{1}(S)}}{\operatorname{argmax}} \sum_{i \in S} f_{i}\left(z_{i, t}, K_{i, t}^{1}\right)-c_{i}\left(e_{i, t}^{1}, \Omega_{i, t}^{1}\right) .
$$

We also define $\left(z_{t}^{\alpha}\right)_{t \in \mathbb{N}}$ as follows:

$$
\forall t \in \mathbb{N}, z_{t}^{\alpha}=\alpha z_{t}^{* 1}+(1-\alpha) z_{t}^{* 0} .
$$

It is straightforward to see that

$$
\sum_{i \in S} \eta_{i, 0}^{\alpha} \leq \breve{y}_{0}=\breve{y}_{0}^{\alpha}
$$

and for each $t \in \mathbb{N}$,

$$
\begin{aligned}
\sum_{i \in S} \eta_{i, t+1}^{\alpha} & =\alpha \sum_{i \in S} \eta_{i, t+1}^{1}+(1-\alpha) \sum_{i \in S} \eta_{i, t+1}^{0} \\
& \leq \alpha \sum_{i \in S}\left(f_{i}\left(z_{i, t}^{* 1}, K_{i, t}^{1}\right)-c_{i}\left(e_{i, t}^{1}, \Omega_{i, t}^{1}\right)\right)+(1-\alpha) \sum_{i \in S}\left(f_{i}\left(z_{i, t}^{* 0}, K_{i, t}^{0}\right)-c_{i}\left(e_{i, t}^{0}, \Omega_{i, t}^{0}\right)\right) .
\end{aligned}
$$

By concavity of $f_{i}$ and $-c_{i}$, we therefore have

$$
\sum_{i \in S} \eta_{i, t+1}^{\alpha} \leq \sum_{i \in S}\left(f_{i}\left(z_{i, t}^{\alpha}, K_{i, t}^{\alpha}\right)-c_{i}\left(e_{i, t}^{\alpha}, \Omega_{i, t}^{\alpha}\right)\right) .
$$

Since for each $t \in \mathbb{N},\left(z_{i, t}^{\alpha}\right)_{i \in S} \in Z^{e_{t}^{\alpha}}(S)$, it follows that

$$
\begin{aligned}
\sum_{i \in S}\left(f_{i}\left(z_{i, t}^{\alpha}, K_{i, t}^{\alpha}\right)-c_{i}\left(e_{i, t}^{\alpha}, \Omega_{i, t}^{\alpha}\right)\right) & \leq \max \left\{\sum_{i \in S} f_{i}\left(z_{i, t}, K_{i, t}^{\alpha}\right)-c_{i}\left(e_{i, t}^{\alpha}, \Omega_{i, t}^{\alpha}\right),\left(z_{i, t}\right)_{i \in S} \in Z^{e_{t}^{\alpha}}(S)\right\} \\
& =\breve{y}_{t+1}^{\alpha} .
\end{aligned}
$$

By combining Eqs 2, 3 and 4, we deduce that for each $t \in \mathbb{N}$,

$$
\sum_{i \in S} \eta_{i, t}^{\alpha} \leq \breve{y}_{t}^{\alpha}
$$

On the other hand, it is clear that

$$
e_{0}^{\alpha} \leq \Omega_{0}
$$

and for each $t \in \mathbb{N}$,

$$
e_{t+1}^{\alpha}=\alpha e_{t+1}^{1}+(1-\alpha) e_{t+1}^{0} \leq \alpha\left(\Omega_{t}^{1}-e_{t}^{1}\right)+(1-\alpha)\left(\Omega_{t}^{0}-e_{t}^{0}\right)=\Omega_{t}^{\alpha}-e_{t}^{\alpha} .
$$

\footnotetext{
${ }^{2}$ It follows from Proposition 1 that both vectors are uniquely defined.
} 
Therefore, from Eqs 5 and 6 , we can conclude that $\left(e_{t}^{\alpha}, \eta_{t}^{\alpha}\right)_{t \in \mathbb{N}}$ is a feasible path. It follows that

$$
\begin{aligned}
V^{\Omega_{0}, K_{0}, \breve{y}_{0}(S)}(S) & \geq \sum_{t \in \mathbb{N}} \delta^{t} v^{\breve{y}_{t}^{\alpha}(S), \eta_{t}^{\alpha}}(S) \\
& =\sum_{t \in \mathbb{N}} \delta^{t}\left(\breve{y}_{t}^{\alpha}(S)-\sum_{i \in S} \eta_{i, t}^{\alpha}\right) \\
& \left.\geq\left(\breve{y}_{0}(S)-\sum_{i \in S} \eta_{i, 0}^{\alpha}\right)+\sum_{t>0} \delta^{t}\left(\sum_{i \in S}\left(f_{i}\left(z_{i, t-1}^{\alpha}, K_{i, t-1}^{\alpha}\right)-c_{i}\left(e_{i, t-1}^{\alpha}, \Omega_{i, t-1}^{\alpha}\right)\right)-\eta_{i, t}^{\alpha}\right)\right) .
\end{aligned}
$$

By strict concavity of $f_{i}$ and $-c_{i}$, we have for each $t>0$ :

$$
\begin{aligned}
\sum_{i \in S}\left(f_{i}\left(z_{i, t-1}^{\alpha}, K_{i, t-1}^{\alpha}\right)-c_{i}\left(e_{i, t-1}^{\alpha}, \Omega_{i, t-1}^{\alpha}\right)\right) & >\alpha \sum_{i \in S}\left(f_{i}\left(z_{i, t-1}^{* 1}, K_{i, t-1}^{1}\right)-c_{i}\left(e_{i, t-1}^{1}, \Omega_{i, t-1}^{1}\right)\right) \\
& +(1-\alpha) \sum_{i \in S}\left(f_{i}\left(z_{i, t-1}^{* 0}, K_{i, t-1}^{0}\right)-c_{i}\left(e_{i, t-1}^{0}, \Omega_{i, t-1}^{0}\right)\right) .
\end{aligned}
$$

Combining Eqs 7 and 8 leads to:

$$
\begin{aligned}
V^{\Omega_{0}, K_{0}, \breve{y_{0}}(S)}(S) & \left.>\alpha\left(\left(\breve{y}_{0}(S)-\sum_{i \in S} \eta_{i, 0}^{1}\right)+\sum_{t>0} \delta^{t} \sum_{i \in S}\left(f_{i}\left(z_{i, t-1}^{* 1}, K_{i, t-1}^{1}\right)-c_{i}\left(e_{i, t-1}^{1}, \Omega_{i, t-1}^{1}\right)\right)-\eta_{i, t}^{1}\right)\right) \\
& \left.+(1-\alpha)\left(\left(\breve{y}_{0}(S)-\sum_{i \in S} \eta_{i, 0}^{0}\right)+\sum_{t>0} \delta^{t} \sum_{i \in S}\left(f_{i}\left(z_{i, t-1}^{* 0}, K_{i, t-1}^{0}\right)-c_{i}\left(e_{i, t-1}^{0}, \Omega_{i, t-1}^{0}\right)\right)-\eta_{i, t}^{0}\right)\right) \\
& =\alpha V^{\Omega_{0}, K_{0}, \breve{y_{0}}(S)}(S)+(1-\alpha) V^{\Omega_{0}, K_{0}, \breve{y}_{0}(S)}(S) \\
& =V^{\Omega_{0}, K_{0}, \breve{y_{0}}(S)}(S) .
\end{aligned}
$$

The strict inequality leads to a contradiction.

\section{Appendix B}

First, let us recall some definitions, notations, and useful results of the cooperative game theory in order to demonstrate Theorem 1 . Let $N$ denote a fixed finite nonempty set with $n$ members, who will be called agents or players. Coalitions of players are nonempty subsets of $N$.

A transferable utility (TU) game on $N$ is a pair $(N, v)$ where $v$ is a mapping $v: 2^{N} \rightarrow \mathbb{R}$ satisfying $v(\emptyset)=0$. We denote by $\mathcal{G}(N)$ the set of all games over $N$. For any coalition $S, v(S)$ represents the worth of $S$, i.e., what coalition $S$ could earn regardless of other players.

A payoff vector is a vector $x \in \mathbb{R}^{N}$ that assigns to agent $i$ the payoff $x_{i}$. A payoff vector is efficient with respect to $(N, v)$ if $\sum_{i \in N} x_{i}=v(N)$; it is coalitionally rational if $\sum_{i \in S} x_{i} \geq v(S)$ for every possible coalition $S$.

The core of $(N, v)$, denoted by $\mathrm{C}(N, v)$, is the set, possibly empty, of efficient and coalitionally rational payoff vectors:

$$
\mathrm{C}(N, v)=\left\{x \in \mathbb{R}^{N}: \forall S \subseteq N, \sum_{i \in S} x_{i} \geq v(S) \text { and } \sum_{i \in N} x_{i}=v(N)\right\} .
$$

The interpretation of the core is that no group of agents has an incentive to split from the grand coalition $N$ and form a smaller coalition $S$ since they collectively receive at least as 
much as what they can obtain for themselves as a coalition. The so-called Bondareva-Shapley theorem (Bondareva, 1963; Shapley, 1967) provides a sufficient and necessary condition under which the core of a TU-game is nonempty. First, we introduce the concept of balanced maps. A balanced map $\lambda: 2^{N} \longrightarrow[0,1]$ is such that:

$$
\lambda(\emptyset)=0, \text { and } \forall i \in N, \quad \sum_{S \ni i} \lambda(S)=1 .
$$

Denote by $\mathbb{B}(N)$ the set of balanced maps over $N$.

Proposition 3. (Bondareva-Shapley Theorem)

For each TU-game $(N, v), \mathrm{C}(N, v) \neq \emptyset$ if and only if for each balanced map $\lambda \in \mathbb{B}(N)$, the following inequality holds:

$$
\sum_{S \subseteq N} \lambda(S) v(S) \leq v(N)
$$

The following proposition is a direct consequence of Corollary 1 in (Gonzalez and Grabisch, 2015).

Proposition 4. If, for each balanced map $\lambda \in \mathbb{B}(N)$ such that $\lambda(N) \neq 1$ the following strict inequality holds:

$$
\sum_{S \subseteq N} \lambda(S) v(S)<v(N)
$$

then $\mathrm{C}(N, v)$ contains an infinity of elements.

\section{Proof of Theorem 1}

Proof. We proceed in two steps.

Step 1: For each $\Omega_{0}(N) \in[0, \bar{\Omega}]^{N}, K_{0}(N) \in[0, \bar{K}]^{N}$ and $\breve{y}_{0}(N) \in[0, \bar{y}]$,

$$
\mathrm{C}\left(N, V^{\Omega_{0}(N), K_{0}(N), \breve{y}_{0}(N)}\right) \neq \emptyset
$$

and contains an infinity of elements.

By Proposition 2, for each nonempty coalition $S \subseteq N$, and each initial stock vector

$$
\left(\Omega_{0}(S), K_{0}(S), \breve{y}_{0}(S)\right) \in[0, \bar{\Omega}]^{S} \times[0, \bar{K}]^{S} \times[0, \bar{y}],
$$

there exists a unique optimal path $\left(e_{t}^{*}(S), \eta_{t}^{*}(S)\right)_{t \in \mathbb{N}}$ to the dynamic programming problem (1). For each nonempty coalition $S \subseteq N$, denote by $\left(\Omega_{t}^{*}(S)\right)_{t \in \mathbb{N}}$ the sequence of resource stock vectors defined by $\Omega_{0}^{*}(S)=\Omega_{0}(S)$ and for each $t \in \mathbb{N}, \Omega_{t+1}^{*}(S)=\Omega_{t}^{*}(S)-e_{t}^{*}(S)$. Denote as well by $\left(K_{t}^{*}(S)\right)_{t \in \mathbb{N}}$ the sequence of capital stock vectors defined by $K_{0}^{*}(S)=K_{0}(S)$ and for each $t \in \mathbb{N}, K_{t+1}^{*}(S)=(1-\tau) K_{t}^{*}(S)+\eta_{t}^{*}(S)$. Finally, denote $\left(\breve{y}_{t}^{*}(S)\right)_{t \in \mathbb{N}}$ the sequence of production flow vectors defined by $\breve{y}_{0}^{*}(S)=\breve{y}_{0}(S)$ and for each $t \in \mathbb{N}$,

$$
\breve{y}_{t+1}^{*}(S)=\max \left\{\sum_{i \in S} f_{i}\left(z_{i, t}(S), K_{i, t}^{*}(S)\right)-c_{i}\left(e_{i, t}^{*}(S), \Omega_{i, t}^{*}(S)\right),\left(z_{i, t}(S)\right)_{i \in S} \in Z^{e_{t}^{*}(S)}(S)\right\} .
$$

Let $\lambda \in \mathbb{B}(N)$ be a balanced system of $N$. If $\lambda(N)=1$, it is clear that

$$
V^{\Omega_{0}(N), K_{0}(N), \breve{y}_{0}(N)}(N)=\sum_{S \subseteq N} \lambda(S) V^{\Omega_{0}(S), K_{0}(S), \breve{y}_{0}(S)}(S) .
$$


Assume $\lambda(N) \neq 1$. We define $\left(e_{t}^{\lambda}(N)\right)_{t \in \mathbb{N}} \in\left(\mathbb{R}_{+}^{N}\right)^{\mathbb{N}}$ and $\left(\Omega_{t}^{\lambda}(N)\right)_{t \in \mathbb{N}} \in\left(\mathbb{R}_{+}^{N}\right)^{\mathbb{N}}$ as follows:

$$
\begin{aligned}
& \forall i \in N, \forall t \in \mathbb{N}, \quad e_{i, t}^{\lambda}(N)=\sum_{S \ni i} \lambda(S) e_{i, t}^{*}(S), \\
& \forall i \in N, \forall t \in \mathbb{N}, \quad \Omega_{i, t}^{\lambda}(N)=\sum_{S \ni i} \lambda(S) \Omega_{i, t}^{*}(S) .
\end{aligned}
$$

Observe that for each $i \in N$ and each $t \in \mathbb{N}$, we have $\Omega_{i, t}^{\lambda}(N) \in[0, \bar{\Omega}]$ since $\sum_{S \ni i} \lambda(S)=1$ and $\Omega_{i, t+1}^{\lambda}(N)=\Omega_{i, t}^{\lambda}(N)-e_{i, t}^{\lambda}(N)$. We also can assert that for each $i \in N$ and each $t \in \mathbb{N}$,

$$
e_{i, t}^{\lambda}(N) \leq \Omega_{i, t}^{\lambda}(N)
$$

We define as well $\left(\eta_{t}^{\lambda}(N)\right)_{t \in \mathbb{N}} \in\left(\mathbb{R}_{+}^{N}\right)^{\mathbb{N}}$ and $\left(K_{t}^{\lambda}(N)\right)_{t \in \mathbb{N}} \in\left(\mathbb{R}_{+}^{N}\right)^{\mathbb{N}}$ as follows:

$$
\begin{aligned}
& \forall i \in N, \forall t \in \mathbb{N}, \quad \eta_{i, t}^{\lambda}(N)=\sum_{S \ni i} \lambda(S) \eta_{i, t}^{*}(S), \\
& \forall i \in N, \forall t \in \mathbb{N}, \quad K_{i, t}^{\lambda}(N)=\sum_{S \ni i} \lambda(S) K_{i, t}^{*}(S) .
\end{aligned}
$$

Observe that for each $i \in N$ and each $t \in \mathbb{N}$, we have $K_{i, t}^{\lambda}(N) \in[0, \bar{K}]$. We set $\breve{y}_{0}^{\lambda}(N)=\breve{y}_{0}(N)$ and for each $t>0$,

$\breve{y}_{t}^{\lambda}(N)=\max \left\{\sum_{i \in N} f_{i}\left(z_{i, t-1}(N), K_{i, t-1}^{\lambda}(N)\right)-c_{i}\left(e_{i, t-1}^{\lambda}(N), \Omega_{i, t-1}^{\lambda}(N)\right),\left(z_{i, t-1}(N)\right)_{i \in N} \in Z^{e_{t-1}^{\lambda}(N)}(N)\right\}$.

Observe that for each $i \in N$ and each $t \in \mathbb{N}$, we have $\breve{y}_{i, t}^{\lambda}(N) \in[0, \bar{y}]$. It is straightforward to see that

$$
\sum_{i \in N} \eta_{i, 0}^{\lambda}(N) \leq \breve{y}_{0}(N)=\breve{y}_{0}^{\lambda}(N)
$$

and for each $t \in \mathbb{N}$,

$$
\begin{aligned}
\sum_{i \in N} \eta_{i, t+1}^{\lambda}(N) & =\sum_{i \in N} \sum_{S \ni i} \lambda(S) \eta_{i, t+1}^{*}(S) \\
& =\sum_{S \subseteq N} \lambda(S) \sum_{i \in S} \eta_{i, t+1}^{*}(S) \\
& \leq \sum_{S \subseteq N} \lambda(S) \breve{y}_{i, t+1}^{*}(S) \\
& =\sum_{S \subseteq N} \lambda(S) \sum_{i \in S}\left(f_{i}\left(z_{i, t}^{*}(S), K_{i, t}^{*}(S)\right)-c_{i}\left(e_{i, t}^{*}(S), \Omega_{i, t}^{*}(S)\right)\right. \\
& =\sum_{i \in N} \sum_{S \ni i} \lambda(S)\left(f_{i}\left(z_{i, t}^{*}(S), K_{i, t}^{*}(S)\right)-c_{i}\left(e_{i, t}^{*}(S), \Omega_{i, t}^{*}(S)\right),\right.
\end{aligned}
$$

where we define, for each $t \in \mathbb{N}$,

$$
z_{t}^{*}(S)=\underset{\left(z_{i, t}(S)\right)_{i \in S} \in Z^{e_{t}^{*}}(S)}{\operatorname{argmax}} \sum_{i \in S} f_{i}\left(z_{i, t}, K_{i, t}^{*}\right)-c_{i}\left(e_{i, t}^{*}, \Omega_{i, t}^{*}\right) .
$$

By concavity of $f_{i}$ and $-c_{i}$, we therefore have

$$
\sum_{i \in N} \eta_{i, t+1}^{\lambda}(N) \leq \sum_{i \in N}\left(f_{i}\left(z_{i, t}^{\lambda}(N), K_{i, t}^{\lambda}(N)\right)-c_{i}\left(e_{i, t}^{\lambda}(N), \Omega_{i, t}^{\lambda}(N)\right)\right)
$$


where $\left(z_{t}^{\lambda}(N)\right)_{t \in \mathbb{N}} \in\left(\mathbb{R}_{+}^{N}\right)^{\mathbb{N}}$ is defined as

$$
\forall i \in N, \forall t \in \mathbb{N}, \quad z_{i, t}^{\lambda}(N)=\sum_{S \ni i} \lambda(S) z_{i, t}^{*}(S) .
$$

The following equalities hold:

$$
\begin{aligned}
\sum_{i \in N} e_{i, t}^{\lambda}(N) & =\sum_{i \in N} \sum_{S \ni i} \lambda(S) e_{i, t}^{*}(S) \\
& =\sum_{S \subseteq N} \lambda(S) \sum_{i \in S} e_{i, t}^{*}(S) .
\end{aligned}
$$

Since $z_{t}^{*}(S) \in Z^{e_{t}^{*}}(S)$, it follows that

$$
\begin{aligned}
\sum_{S \subseteq N} \lambda(S) \sum_{i \in S} e_{i, t}^{*}(S) & =\sum_{S \subseteq N} \lambda(S) \sum_{i \in S} z_{i, t}^{*}(S) \\
& =\sum_{i \in N} \sum_{S \ni i} \lambda(S) z_{i, t}^{*}(S) \\
& =\sum_{i \in N} z_{i, t}^{\lambda}(N),
\end{aligned}
$$

from which we deduce that $z_{t}^{\lambda}(N) \in Z^{e_{t}^{\lambda}(N)}(N)$ for each $t \in \mathbb{N}$. It follows that

$$
\begin{aligned}
\sum_{i \in N}\left(f_{i}\left(z_{i, t}^{\lambda}(N), K_{i, t}^{\lambda}(N)\right)-c_{i}\left(e_{i, t}^{\lambda}(N), \Omega_{i, t}^{\lambda}(N)\right)\right) & \leq \max \left\{\sum_{i \in N} f_{i}\left(z_{i, t}(N), K_{i, t}^{\lambda}(N)\right)\right. \\
& \left.-c_{i}\left(e_{i, t}^{\lambda}(N), \Omega_{i, t}^{\lambda}(N)\right),\left(z_{i, t}(N)\right)_{i \in N} \in Z^{e_{t}^{\lambda}(N)}(N)\right\} \\
& =\breve{y}_{t+1}^{\lambda}(N) .
\end{aligned}
$$

By combining Eqs 11, 13 and 14, we deduce that for each $t \in \mathbb{N}$,

$$
\sum_{i \in N} \eta_{i, t}^{\lambda}(N) \leq \breve{y}_{t}^{\lambda}(N)
$$

We can conclude from Eqs 10 and 15 that $\left(e_{t}^{\lambda}(N), \eta_{t}^{\lambda}(N)\right)_{t \in \mathbb{N}}$ is a feasible path.

By definition of $\breve{y}_{t}^{\lambda}(N)$ for each $t \in \mathbb{N}$, and since $z_{t}^{\lambda}(N) \in Z_{t}^{e_{t}^{\lambda}(N)}(N)$ for each $t \in \mathbb{N}$, we have

$$
\breve{y}_{t+1}^{\lambda}(N) \geq \sum_{i \in N}\left(f_{i}\left(z_{i, t}^{\lambda}(N), K_{i, t}^{\lambda}(N)\right)-c_{i}\left(e_{i, t}^{\lambda}(N), \Omega_{i, t}^{\lambda}(N)\right)\right) .
$$

The hypothesis of strict concavity ensures that

$\sum_{i \in N}\left(f_{i}\left(z_{i, t}^{\lambda}(N), K_{i, t}^{\lambda}(N)\right)-c_{i}\left(e_{i, t}^{\lambda}(N), \Omega_{i, t}^{\lambda}(N)\right)\right)>\sum_{i \in N} \sum_{S \ni i} \lambda(S)\left(f_{i}\left(z_{i, t}^{*}(S), K_{i, t}^{*}(S)\right)-c_{i}\left(e_{i, t}^{*}(S), \Omega_{i, t}^{*}(S)\right)\right)$

because for each $i \in N$ and $t \in \mathbb{N}, z_{i, t}^{\lambda}(N)$ (resp. $\left.K_{i, t}^{\lambda}(N), e_{i, t}^{\lambda}(N), \Omega_{i, t}^{\lambda}(N)\right)$ is a convex combination of $\left(z_{i, t}^{*}(S)\right)_{S \ni i}\left(\operatorname{resp} .\left(K_{i, t}^{*}(S)\right)_{S \ni i},\left(e_{i, t}^{*}(S)\right)_{S \ni i},\left(\Omega_{i, t}^{*}(S)\right)_{S \ni i}\right)$.

Combining Eqs. 16 and 17, we have:

$$
\begin{aligned}
\breve{y}_{t+1}^{\lambda}(N) & >\sum_{i \in N} \sum_{S \ni i} \lambda(S)\left(f_{i}\left(z_{i, t}^{*}(S), K_{i, t}^{*}(S)\right)-c_{i}\left(e_{i, t}^{*}(S), \Omega_{i, t}^{*}(S)\right)\right) \\
& =\sum_{S \subseteq N} \lambda(S) \sum_{i \in S}\left(f_{i}\left(z_{i, t}^{*}(S), K_{i, t}^{*}(S)\right)-c_{i}\left(e_{i, t}^{*}(S), \Omega_{i, t}^{*}(S)\right)\right) \\
& =\sum_{S \subseteq N} \lambda(S) \breve{y}_{t+1}^{*}(S) .
\end{aligned}
$$


Since $\left(e_{t}^{\lambda}(N), \eta_{t}^{\lambda}(N)\right)_{t \in \mathbb{N}}$ is a feasible path, and that $\Omega_{0}^{\lambda}(N)=\Omega_{0}(N), K_{0}^{\lambda}(N)=K_{0}(N)$ and $\breve{y}_{0}^{\lambda}(N)=\breve{y}_{0}(N)$, the definition of $V^{\Omega_{0}(N), K_{0}(N), \breve{y}_{0}(N)}(N)$ ensures that

$$
V^{\Omega_{0}(N), K_{0}(N), \breve{y}_{0}(N)}(N) \geq \sum_{t \in \mathbb{N}} \delta^{t}\left(\breve{y}_{t}^{\lambda}(N)-\sum_{i \in N} \eta_{i, t}^{\lambda}(N)\right) .
$$

Moreover, observe that

$$
\breve{y}_{0}(N)=\sum_{S \subseteq N} \lambda(S) \breve{y}_{0}(S)
$$

Indeed,

$$
\begin{aligned}
\sum_{S \subseteq N} \lambda(S) \breve{y}_{0}(S) & =\sum_{S \subseteq N} \lambda(S) \sum_{i \in S} \breve{y}_{i, 0} \\
& =\sum_{i \in N} \sum_{S \ni i} \lambda(S) \breve{y}_{i, 0} \\
& =\sum_{i \in N} \breve{y}_{i, 0} \sum_{S \ni i} \lambda(S) \\
& =\sum_{i \in N} \breve{y}_{i, 0}
\end{aligned}
$$

Using Eqs. 19, 12 and 18, we obtain

$$
\begin{aligned}
V^{\Omega_{0}(N), K_{0}(N), \breve{y}_{0}(N)}(N) & =\left(\breve{y}_{0}(N)-\sum_{i \in N} \eta_{0}^{\lambda}(N)\right)+\sum_{t>0} \delta^{t}\left(\breve{y}_{t}^{\lambda}(N)-\sum_{i \in N} \eta_{i, t}^{\lambda}(N)\right) \\
& >\left(\sum_{S \subseteq N} \lambda(S) \breve{y}_{0}(S)-\sum_{S \subseteq N} \lambda(S) \sum_{i \in S} \eta_{i, 0}^{*}(S)\right) \\
& +\sum_{t>0} \delta^{t}\left(\sum_{S \subseteq N} \lambda(S) \breve{y}_{t}^{*}(S)-\sum_{S \subseteq N} \lambda(S) \sum_{i \in S} \eta_{i, t}^{*}(S)\right) \\
& =\sum_{S \subseteq N} \lambda(S) \sum_{t \in \mathbb{N}} \delta^{t}\left(\breve{y}_{t}^{*}(S)-\sum_{i \in S} \eta_{i, t}^{*}(S)\right) \\
& =\sum_{S \subseteq N} \lambda(S) V^{\Omega_{0}(S), K_{0}(S), \breve{y}_{0}(S)}(S) .
\end{aligned}
$$

The strict inequality holds by strict concavity and since $\lambda(N) \neq 1$.

By Proposition 3 stating the Bondareva-Shapley theorem, $\mathrm{C}\left(N, V^{\Omega_{0}(N), K_{0}(N), \breve{y}_{0}(N)}\right) \neq \emptyset$ and by Proposition $4, \mathrm{C}\left(N, V^{\Omega_{0}(N), K_{0}(N), \breve{y}_{0}(N)}\right)$ contains an infinity of elements.

\section{Step 2: Existence and non uniqueness of the solution.}

Step 1 states that for each state $(\Omega, K, \breve{y})$, the core $\mathrm{C}\left(N, V^{\Omega, K, \breve{y}}\right)$ is nonempty. Hence, for each initial state $(\Omega, K, \breve{y})$ we can build a sequence $\left(\left(X_{i, t}(\Omega, K, \breve{y})\right)_{i \in N}\right)_{t \in \mathbb{N}}$ - or simply $\left(\left(X_{i, t}\right)_{i \in N}\right)_{t \in \mathbb{N}}$ - such that for each $t \in \mathbb{N}$ :

(i) $\sum_{i \in N} X_{i, t}=V^{\Omega_{t}^{*}(N), K_{t}^{*}(N), \breve{y}_{t}^{*}(N)}(N)$,

(ii) $\sum_{i \in S} X_{i, t} \geq V^{\Omega_{S, t}^{*}(N), K_{S, t}^{*}(N), \breve{y}_{S, t}^{*}(N)}(S)$,

where $\left(\Omega_{t}^{*}(N), K_{t}^{*}(N), \breve{y}_{t}^{*}(N)\right)_{t \in \mathbb{N}}$ is the sequence of state vectors associated with the optimal extraction and investment path of $N$ when the initial state is $(\Omega, K, \breve{y})$.

Let the instantaneous allocation path for each country be $\left(x_{i, t}(\Omega, K, \breve{y})\right)_{i \in N, t \in \mathbb{N}}$ - or simply $\left(x_{i, t}\right)_{i \in N, t \in \mathbb{N}}$ - defined for each initial state $(\Omega, K, \breve{y})$ such that for each $t \in \mathbb{N}, x_{i, t}=X_{i, t}-\delta X_{i, t+1}$.

Let us first show that $\left(x_{i, t}\right)_{i \in N, t \in \mathbb{N}}$ is an international distribution policy. For each $t \in \mathbb{N}$, 


$$
\begin{aligned}
\sum_{i \in N} x_{i, t} & =\sum_{i \in N} X_{i, t}-\delta \sum_{i \in N} X_{i, t+1} \\
& =V^{\Omega_{t}^{*}(N), K_{t}^{*}(N), \breve{y}_{t}^{*}(N)}(N)-\delta V^{\Omega_{t+1}^{*}(N), K_{t+1}^{*}(N), \breve{y}_{t+1}^{*}(N)}(N)
\end{aligned}
$$

and the Bellman equation ensures that:

$$
V^{\Omega_{t}^{*}(N), K_{t}^{*}(N), \breve{y}_{t}^{*}(N)}(N)=v^{\breve{y}_{t}^{*}(N), \eta_{t}^{*}(N)}(N)+\delta V^{\Omega_{t+1}^{*}(N), K_{t+1}^{*}(N), \breve{y}_{t+1}^{*}(N)}(N) .
$$

Therefore, we have for each $t \in \mathbb{N}$,

$$
\sum_{i \in N} x_{i, t}=v^{\breve{y}_{t}^{*}(N), \eta_{t}^{*}(N)}(N) .
$$

Let us now prove that $\left(x_{i, t}\right)_{i \in N, t \in \mathbb{N}}$ is an undominated policy. For each $T \in \mathbb{N}$, we have

$$
\begin{aligned}
\sum_{i \in S} \sum_{t=0}^{\infty} \delta^{t} x_{i, t+T} & =\sum_{i \in S} \sum_{t=0}^{\infty}\left(\delta^{t} X_{i, t+T}-\delta^{t+1} X_{i, t+T+1}\right) \\
& =\sum_{i \in S} X_{i, T} \\
& \geq V^{\Omega_{S, T}^{*}(N), K_{S, T}^{*}(N), \breve{y}_{S, T}^{*}(N)}(S) .
\end{aligned}
$$

We can therefore conclude that the set $\mathcal{C}(\Omega, K, \breve{y})$ of undominated policies when the initial state is $(\Omega, K, \breve{y})$ is nonempty. Since we established in Step 1 that for each state $(\Omega, K, \breve{y})$, $\mathrm{C}\left(N, V^{\Omega, K, \breve{y}}\right)$ contains an infinity of elements, we deduce by construction of $\left(x_{i, t}\right)_{i \in N, t \in \mathbb{N}}$ that $\mathcal{C}(\Omega, K, \breve{y})$ also contains an infinity of elements. 